\title{
A Review of Watermarking Requirements, Techniques, Documents, Human Perception and Applications for Medical Images
}

\author{
M.Manivannan ${ }^{1}$, Dr.G.Suseendran ${ }^{2}$ \\ ${ }^{1}$ Research Scholar, Bharathiar University, Coimbatore, India \\ ${ }^{2}$ Assistant Professor, School of Computing Science, Vels University, Chennai, India \\ Email: mmanivannan.s@gmail.com ${ }^{1}$, suseendar_1234@yahoo.com ${ }^{2}$
}

\section{Abstract}

The objective of this paper is to separate the audit business related in the field of watermarking concentrating solely on restorative picture watermarking. Medicinal imaging has affected decidedly the social insurance framework around the globe by helping specialists perform visual diagnostics of the human body. One of the broadly utilized applications is to shield the patient's therapeutic history from unapproved individuals. It implants the watermark like patient's data and specialist's mark in host's restorative picture for telemedicine applications. It can be additionally utilized for confirmation if tolerant lost his/her picture. Watermarking in therapeutic pictures is regularly utilized for content verification, viable information circulation and administration, stockpiling, security, safe filing, controlled get to recovery and captioning. Most of the works are on the alter recognition of the pictures and inserting of the Electronics Patient Record (EPR) information in the medicinal.

Keywords: Digital Watermarking, Electronic Patient Record (EPR), Medical Image Watermarking (MIW), Image Retrieval.

\section{Introduction}

Presently a day's transmission of therapeutic pictures among doctor's facilities over web is a typical practice. It should be possible for some reasons which incorporate video chats, tele-finding, tele- conference administrations etc. Guaranteeing the security of traded therapeutic information has turned into a noteworthy risk. The restorative picture security can be founded on specific components like (a) Authentication (b) Integrity (c) Confidentiality [1][18]. It guarantees that the substance that is conveying is the very same individual or association that it cases to be. It can be utilized as a proof to guarantee that the data is that of the correct patient. It guarantees that the information acquired at the flip side are same as what has been sent by the approved sender (i.e., no inclusion, adjustment, replay or cancellation has happened). The information can be shielded from unlawful exposure, i.e., just the allowed client can have the privilege to utilize the data. As depicted in figure 1 , reliability is extendable to traceability when it becomes possible to trace the information along its distribution. Traceability allows systematic information content validation, which is aimed at trustiness and quality control: actuality (precise interest of the information at a given instant) and reliability. 


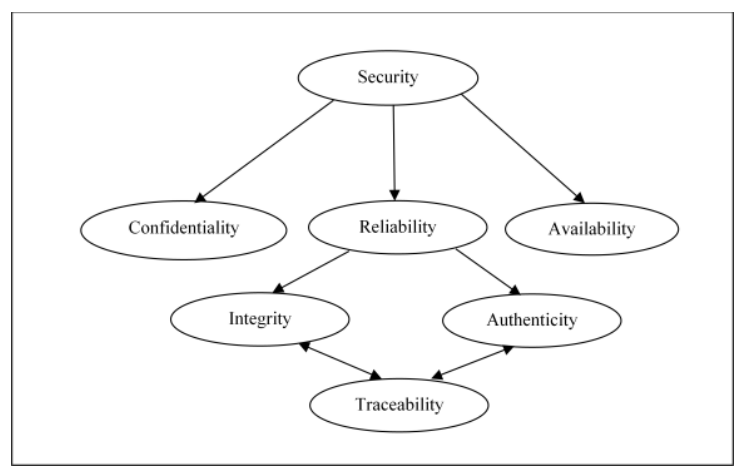

Fig 1: Medical information security components

Watermarking in restorative pictures is another range of research and a few works around there have been all inclusive as of late.

\section{Watermarking Techniques on Medical Image Watermarking}

MIW techniques can be classified into two domains: Spatial domain watermarking and Frequency domain watermarking. The whole process of MIW techniques is shown in figure 2.

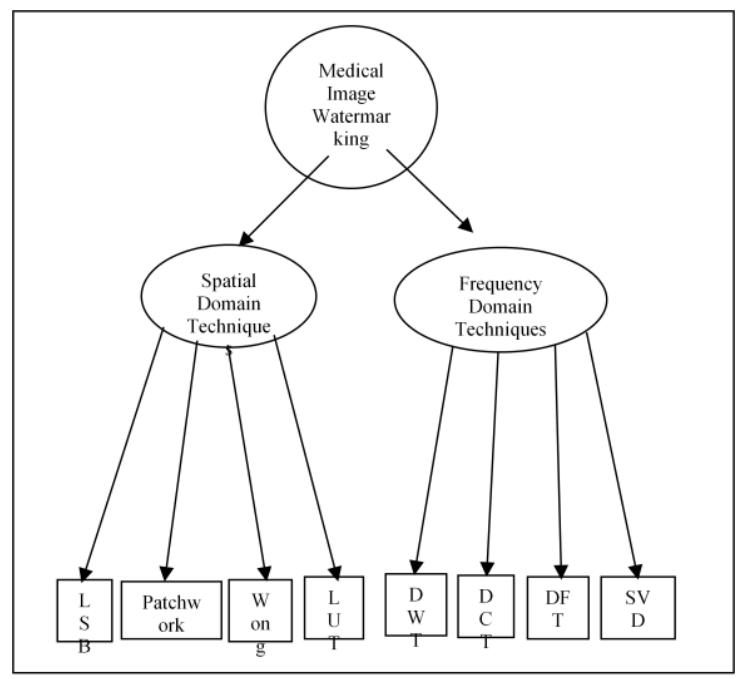

Fig 2: Medical Image Watermarking Techniques

\subsection{Spatial domain watermarking}

In spatial area system the watermark inserting is accomplished by specifically adjusting the pixel estimations of the host picture. The most regularly utilized strategy in the spatial space method is the minimum noteworthy piece (LSB).

\subsubsection{Least Significant Bit Coding (LSB)}

LSB [2] coding is one of the soonest strategies for picture watermarking. In this technique the inclusion of information is just done in the low-arrange bits of the picture. For a picture coded on 8 bits, the adjustment of the LSB causes a variety of the dim level of $1 / 256$. This adjustment by and by is undetectable. In the event that this strategy gets great outcomes for what is the imperceptibility, it is not acceptable for the power.

\subsubsection{Patchwork Technique}

Bender et al. [3] proposed watermarking plan in view of factual technique called interwoven. In this system, $\mathrm{n}$ sets of picture focuses (a, b) are haphazardly picked. The picture information in "an" is helped while that in " $b$ " is obscured in a similar scale. Test comes about demonstrate that this calculation is straightforward and simple, indicating sensibly high imperviousness to generally changes. However there are a few restrictions, for example, greatly low implanted information rate and thus this procedure is helpful to low piece rate applications as it were.

\subsubsection{Wong Technique}

The algorithm of Wong [4] permits watermarking the picture in the spatial space, it embeds two sorts of particular information at the level of the LSB: the first is a double logo, to recognize the proprietor of the picture, and the other a synopsis of the picture got from a hash work MD5 [5]. It is to partition the picture and the logo by squares of $\mathrm{i} * \mathrm{j}$ pixels and ascertain the outline of each piece of the picture. And after that, the principal bit of synopsis acquired are then added by a selective or to the piece of the logo which 
will be then embed at the level of the LSB of the square of the picture to watermark.

\subsubsection{Look up Table (LUT) Technique}

Yeung [6] inserts the watermark by using a LUT for each pixel in the photo, the LUT grants to get the looking at twofold regard. If it is vague to the estimation of the bit to be inserted, the pixel of the photo is not changed but instead if the regard is phenomenal, the pixel is adjusted until the right regard.

\subsection{Frequency Domain Watermarking}

This system is generally called Transform space. In this strategy estimations of particular frequencies are changed from their extraordinary regards. There are diverse methodologies which are used as a piece of progress strategy, for instance, DWT, DCT, DFT and SVD.

\subsubsection{Discrete Wavelet Technique (DWT)}

Discrete Wavelet change (DWT) [7] is a scientific apparatus for falling apart the photo. The change relies upon little waves called wavelet of moving repeat. The wavelets change fall apart the photo into three heading level, vertical and corner to corner.

\subsubsection{Discrete Cosine Transform (DCT)} Discrete Cosine Transform (DCT) [8] addresses data as repeat rather than an ampleness space. DCT watermarking techniques are energetic diverged from spatial zone frameworks. The watermark is introduced into the coefficients of the inside repeat, so the deceivability of picture won't get affected and the watermark won't be ousted by any kind of ambush.

\subsubsection{Discrete Fourier Transform (DFT)}

Discrete Fourier Transform (DFT) [9] offers control against geometric attacks like turn, scaling, altering, and understanding et cetera. DFT separates a photo in sine and cosine shape. The DFT based watermark embeddings strategies are secluded in two sorts: one is the direct embedding and the other one is the arrangement based introducing.

According to the direct embeddings procedure the watermark is introduced by changing DFT size and stage coefficients. The arrangement based embedding methodology introduces the possibility of formats. A configuration is structure which is embedded in the DFT region to evaluate the change factor. Once the photo encounters a change this configuration is looked to resynchronize the photo, and subsequently the pointer is used to remove the introduced spread range watermark.

\subsubsection{Singular value decomposition (SVD)}

Particular esteem decay (SVD) [10]is a champion among the most a significant part of the time used gadgets of straight factor based math with a couple of utilizations in picture weight, watermarking and diverse scopes of banner taking care of. In watermarking a huge bit of the SVD methods are used to change the singular estimations of host picture by the specific estimations of watermark. By alluding different papers we arranged after table I which gives synopsis of various watermarking systems with favorable circumstances and burdens [11-13]. 


\begin{tabular}{|c|c|c|}
\hline $\begin{array}{c}\text { Medical } \\
\text { ImageWatermarking } \\
\text { Techniques }\end{array}$ & Advantages & Disadvantages \\
\hline LSB & $\begin{array}{l}\text { 1. Easy to implement and understand. } \\
\text { 2. Low degradation of image quality. } \\
\text { 3. High perceptual transparency. }\end{array}$ & $\begin{array}{l}\text { 1. It lacks basic robustness. } \\
\text { 2. Vulnerable to noise. } \\
\text { 3. Vulnerable to cropping, } \\
\text { scaling. }\end{array}$ \\
\hline Patchwork & $\begin{array}{l}\text { High level of robustness against } \\
\text { most type of attacks. }\end{array}$ & $\begin{array}{l}\text { It can hide only a very small } \\
\text { amount of information. }\end{array}$ \\
\hline Wong & $\begin{array}{l}\text { Watermarking the image in the } \\
\text { spatial domain. }\end{array}$ & $\begin{array}{l}\text { Watermarking the image doesn't } \\
\text { work in the frequency domain. }\end{array}$ \\
\hline LUT & It has high speed. & It has high memory usage. \\
\hline DCT & $\begin{array}{l}\text { The watermark is embedded into } \\
\text { the coefficients of the middle } \\
\text { frequency, so the visibility of } \\
\text { image will not get affected and the } \\
\text { watermark will not be removed by } \\
\text { any kind of attack. }\end{array}$ & $\begin{array}{l}\text { 1. Block wise DCT destroys the } \\
\text { invariance properties of the } \\
\text { system. } \\
\text { 2. Certain higher frequency } \\
\text { components tend to be } \\
\text { suppressed during the } \\
\text { quantization step. } \\
\text { 3. DCT technique doesn't work } \\
\text { with scaling attacks. }\end{array}$ \\
\hline DWT & $\begin{array}{l}\text { 1. Allows good localization both in } \\
\text { time and spatial frequency domain. } \\
\text { 2. Higher compression ratio which is } \\
\text { relevant to human perception. } \\
\text { 3. More robust to cropping. } \\
\text { 4. It has multi resolution } \\
\text { characteristics and is hierarchical. } \\
\text { 5. DWT has effective also in } \\
\text { structural attacks. }\end{array}$ & $\begin{array}{l}\text { 1. Cost of computing may be } \\
\text { higher. } \\
\text { 2. Longer compression time. } \\
\text { 3. Noise/blur near edges of } \\
\text { images or video frames. }\end{array}$ \\
\hline DFT & $\begin{array}{l}\text { DFT is rotation, scaling and } \\
\text { translation (RST) invariant. Hence it } \\
\text { can be used to recover from } \\
\text { geometric distortions. }\end{array}$ & $\begin{array}{l}\text { 1. Complex implementation } \\
\text { 2. Cost of computing may be } \\
\text { higher. }\end{array}$ \\
\hline SVD & $\begin{array}{l}\text { 1. SVD of a matrix is a powerful } \\
\text { technique in matrix computations. } \\
\text { 2. SVD is used as a method for } \\
\text { noise reduction. }\end{array}$ & $\begin{array}{l}\text { 1. Cost of computing may be } \\
\text { higher. } \\
\text { 2. SVD operates on a fixed } \\
\text { matrix, hence it is not amenable } \\
\text { to problems that require adaptive } \\
\text { algorithms. }\end{array}$ \\
\hline
\end{tabular}




\section{Related to Document on Medical Image Watermarking}

According to the type of document, the medical image watermarks can be divided into 5 different types: Image, Audio, Video, Text and Graphic watermarking.

Picture is watermarked utilizing a primary picture, unique picture lastly picture is then watermarked. This is utilized to conceal the extraordinary data into the picture and to later recognize and concentrate that exceptional data for the creator's proprietorship [14].This application area is one of the most popular and happening issue due to teleconsultation [14].This includes watermark in the video stream to order video applications. This technique requires continuous extraction and vigor for pressure [14].

This adds watermark to the PDF, DOC and other content document to keep the progressions made to content. The watermark is embedded in the text style shape and the space amongst characters and line spaces [14].It implants the watermark to $2 \mathrm{D}$ or $3 \mathrm{D}$ PC produced illustrations to show the copyright [14].

\section{Related to Human Perception on Medical Image Watermarking}

$\mathrm{s}$ indicated by the human discernment, the medicinal picture watermarks can be partitioned into 2 distinct sorts: Visible and Invisible watermark. The watermark is unmistakable that can be a content or a logo. It is utilized to distinguish the proprietor [15].The watermark is installed into the picture such that it can't be seen by human eye. It is utilized to secure the picture verification and furthermore keep it from being duplicated. Imperceptible watermark can be additionally grouped into three types: Robust Watermark means to implant data in a document that can't be effortlessly demolished. They are intended to oppose any controls that might be experienced. All applications where security is the fundamental issue utilize hearty watermark. They are composed with low heartiness. It is utilized to check the honesty of articles They are separated as per the mystery prerequisites for the key used to install and recover watermarks. If the main picture is not known in the midst of the revelation strategy then it is known as an open or an outwardly hindered watermark and if the primary picture is referred to it is known as a non-stun watermark or a private watermark

\section{Applications of Medical Image Watermarking}

Watermarking advances is connected in each medicinal applications while security and proprietor recognizable proof is required [16]. Therapeutic media and reports likewise carefully checked, having the data of patient and the meeting specialists. These watermarks can be both unmistakable and imperceptible. This watermarking helps specialists and restorative applications to confirm that the reports are not altered by unlawful means. Authentication is the procedure of distinguish that the got substance or information ought to be correct as it was sent.

\section{Requirements of Medical Image Watermarking}

The fundamental necessities in restorative picture watermarking are firmly identified with its motivation of utilizations, diverse application has distinctive requests.

Cox et al. [14] characterize straightforwardness or devotion as "perceptual closeness between the first and the watermarked variants of the cover work". Presentation of unmistakable mutilations ought not be there in watermarked picture in light of the fact 
that if such bends exist it decreases the business estimation of the picture.

Cox et al. [17] characterize heartiness as the "capacity to identify the watermark after normal flag handling operations". At the end of the day, installed watermark ought to be difficult to recognize, expel, or supplant by unlawful sources without corrupting the nature of the substance to low to such an extent that it ends up plainly unusable.

Cox et al. [17] characterize limit or information payload as "the quantity of bits a watermark encodes inside a unit of time or work". To speak to the uniqueness, the watermark ought to have the capacity to convey that much data that gives some important message.

A strong watermarking strategy is required with the goal that the message can't be perused by the interloper. It is the method for cross-check whether the substance has truly been communicated or not on the planned timetable. To check when and where each clasp appears there are computerized observing stations for getting communicates and pay special mind to the implanted watermarks [17].

This is the most widely recognized use of computerized watermarking. At whatever point any sort of changes is done to the advanced substance the watermark gets demolished such a watermark is called delicate watermark. This sort of watermark is utilized as a part of alter recognition. On the off chance that the removed watermark is mistaken, one might say that the first substance is altered. Significance of this application is by the association of very delicate information like restorative imaging or satellite imaging [18]. It is one of the soonest uses of watermarking, or we can state information stowing away to send mystery messages [18].
As no pernicious assaults are utilized, the substance has simply to go by normal flag preparing so there is no need of advanced watermarking procedures [19]. It is required when confirmations are to be introduced to an expert. The point is to identify a few adjustments done to the substance. The inserted watermark ought to be special and difficult to identify or evacuate by the assailant. Some watermarking methods utilize altered district limitation to identify the tempered locales of the modified substance [19].

\section{Attacks of Medical Image Watermarking}

There are different conceivable pernicious deliberate or accidental assaults that a watermarked protest is probably going to subject to. The accessibility of extensive variety of picture preparing virtual products made it conceivable to perform assaults on the strength of the watermarking frameworks. The point of these assaults is keep the watermark from playing out its proposed reason [12].

Evacuation assaults mean to expel the watermark information from the watermarked question. Such assaults abuse the way that the watermark is normally an added substance clamor flag exhibit in the host flag. Impedance assaults are those which add extra commotion to the watermarked question. Lossy pressure, quantization, intrigue, denoising, remodulation, averaging, and clamor storm are a few cases of this classification of assaults. All controls that influence the geometry of the picture, for example, flipping, turn, trimming, and so on ought to be perceptible. A trimming assault from the right-hand side and the base of the picture is a case of this assault.

A low ignore separating is done the watermarked picture and it brings about a distinction delineate of commotion. The 
falsification assaults that outcome in protest inclusion and erasure, scene foundation changes are altogether equivalent to substitution.In specific, if the watermarking calculation is known, an aggressor can additionally endeavor to perform adjustments to render the watermark invalid or to evaluate and alter the watermark. For this situation, we discuss an assault on security. The watermarking calculation is viewed as secure if the inserted data can't be demolished, identified or manufactured. The convention assaults do neither go for decimating the implanted data nor at incapacitating the discovery of the installed data (deactivation of the watermark).

Instead of that, they exploit semantic shortfalls of the watermarks usage. Thus, a strong watermark must not be invertible or to be duplicated. A duplicate assault, for instance, would go for replicating a watermark from one media into another without information of the mystery key. Cryptographic assaults manage the breaking of the security. For instance, finding the mystery watermarking key utilizing comprehensive animal compel strategy is a cryptographic assault [20].

\section{Conclusion}

In this paper, I have presented some essential ideas in restorative picture watermarking, including its establishment, prerequisites, applications and also the examination between therapeutic picture watermarking strategies. In this paper, we attempted our best to give the entire data of therapeutic picture watermarking which will offer profit to new scientists to get the most extreme mindfulness about this domain.So in future we can chip away at new calculations of watermarking consolidate with different strategies that may decrease or evacuate the disservices of past calculations.

\section{References}

[1] Umamageswari, A., and G. R. Suresh. "Performance Analysis of Secure Medical Image Communication with Digital Signature and Reversible Watermarking." ictact journal on image and video processing 4.01: 647-651, 2013.

[2] Mitchell D. Swanson, Mei Kobayashi, Ahmed H. Tewfik, Multimedia dataembedding and watermarking technologies in Proceedings of the IEEE, vol. 86(6), p. 1064-1087, June 1998.

[3] W. Bender, D. Gruhl, N. Morimoto, Techniques for Data Hiding in Proceedings of the SPIE Conference on Storage and Retrieval for Image and Video Databases III, San Jose, CA, vol. 2420, p. 164-173, February 1995.

[4] WongP.W., A Public Key Watermark for Image Verification and Authentication Proceedings of the Int. Conf. Im. Proc, vol. I, p. 155-459, 1998.

[5] Mehmet U. Celik, Gaurav Sharma, A Hierarchical Image Authentication Watermark With Improved Localization And Security in Proceedings of the IEEE International Conference on Image Processing, vol. II, p. 502-505, Oct 2001.

[6] M. Yeung, F. Mintzer, An Invisible Watermarking Technique for Image Verification in Proceedings of the IEEE ICIP, Santa Barbara, Oct 1997.

[7] Vaishali S. Jabade, Dr. Sachin R. Gengaje "Literature Review of Wavelet Based Digital Image Watermarking Techniques", International Journal of Computer Applications (0975 - 8887) Volume 31- No.1, October 2011.

[8] Prabhishek Singh, R S Chadha, "A Survey of Digital Watermarking 
Techniques, Applications and Attacks", International Journal of Engineering and Innovative Technology (IJEIT) Volume 2, Issue 9, March 2013.

[9] V. M. Potdar, S. Han and E. Chang, "A Survey of Digital Image Watermarking Techniques", 2005 3rd IEEE International Conference on Industrial Informatics (INDIN).

[10] K. J. Kavitha, M. Reshma, "A Survey on Digital Watermarking on Medical Images", International Journal of Engineering of Advanced Computer Research (IJEACR) Volume 3, Issue 13, December 2013.

[11] "Watermarking Techniques Spatial Domain Digital Rights Seminar @,", Mahmoud El-Gayyar, Media Informatics University of Bonn Germany.

[12] Prabhishek Singh, R S Chadha, "A Survey of Digital Watermarking Techniques, Applications and Attacks", International Journal of Engineering and Innovative Technology (IJEIT), Volume 2, Issue 9, March 2013.

[13] Jiang Xuehua, Digital Watermarking and Its Application in Image Copyright Protection, 2010 International Conference on Intelligent Computation Technology and Automation.

[14] Rashmi Soni, M.K. Gupta, "Comparative Analysis of Digital Watermarking Based on Embedding and Extraction Technique", International Journal of Computer Engineering \& Technology (IJCET) Volume 4, Issue 6, December 2013.

[15] Sasmita Mishra, AmitavMahapatra,Pranati Mishra, "A Survey on Digital Watermarking Techniques", International Journal of Computer Science and Information
Technologies, ISSN:0975-9646, Vol. 4 , 2013, 451-456.

[16] Mei Jiansheng, Li Sukang, "A Digital Watermarking Algorithm Based On DCT and DWT", Proceedings of the 2009 International Symposium on Web Information Systems and Applications (WISA ${ }^{\text {ee }}$ 9) Nanchang, P. R. China, May 22-24, 2009, pp. 104-107.

[17] I. J. Cox, M. L. Miller, J. A. Bloom, and C. Honsinger, Digital watermarking, vol. 53. Springer, 2002.

[18] I. J. Cox, M. L. Miller, and J. A. Bloom, "Watermarking applications and their properties," in itcc, 2000, pp. 6-10.

[19] M. K. Samee, "Robust watermarking and its applications to communication problems," 2012.

[20] G. Coatrieux, L. Lecornu, Members, IEEE, Ch. Roux, Fellow, IEEE, B. Sankur, Member, IEEE, $\|$ A Review of digital image watermarking in health care. 